\title{
Factor Analysis of Predicting Cardiovascular Death in the Remote Period after Myocardial Revascularization
}

\author{
Ekaterina S. Levitskaya ${ }^{1 *}, \mathrm{PhD}$; Mikhail M. Batiushin ${ }^{1}, \mathrm{PhD}, \mathrm{ScD}$; \\ Elena O. Golovinova'; Valentina V. Gul'chenko'; \\ Vladimir A. Chistjakov ${ }^{1,2}$; Dmitriy G. Pasechnik ${ }^{1}$ \\ ${ }^{1}$ Rostov State Medical University, ${ }^{2}$ Southern Federal University \\ Rostov-on-Don, Russia
}

\begin{abstract}
The aim of the present study was to analyze the impact of traditional and renal risk factors (RFs) on the probability of cardiovascular death in CHD patients in the remote period after myocardial revascularization (MR).

Materials and Methods: The present study included 90 CHD patients ( 80 men and 10 women; mean age $56.1 \pm 0.9$ years) with indications for MR. The prevalence of major cardiovascular RFs (old age, gender, duration of CHD, arterial hypertension, diabetes mellitus, another localization of atherosclerotic lesions, and the presence and duration of smoking) and the main echocardiographic parameters and the parameters of renal function (MAU and GFR) were assessed. Fatal cardiovascular outcomes were the only endpoint of the study.

Results: Cardiovascular death (CVD) occurred in 10/12.3\% patients. The studied RFs, such as the patient's age, duration of smoking, and presence of angina with low tolerance to physical stress, had a significant impact on the probability of death in CHD patients. Risk of CVD (rCVD) over a long-term period increases by $18.1 \%$ in patients with elevated levels of total cholesterol, by $16.2 \%$ in patients with stable angina pectoris class III, by $50.5 \%$ in patients with atherosclerotic lesions of lower limb and cerebral arteries, and by $69.3 \%$ in patients suffering from overweight. Left atrial size, LVPWT, and LVMI were also significant predictors of adverse cardiovascular prognosis. The increase in the number of coronary arteries with clinically significant stenosis, including subtotal narrowing of the vessel lumen, increases rCVD in the long-term period. The important role of a highly reliable level of glucose in the urine for the risk score was found. The presence of CKD stage 3 and the impaired GFR also significantly increased rCVD.

Conclusion: Our data demonstrate a high medical and social importance of a comprehensive and integrated analysis not only of traditional RFs, but also of markers of renal dysfunction in risk stratification of cardiovascular prognosis in CHD patients.
\end{abstract} (Int J Biomed. 2015;5(3):117-122.)

Keywords: coronary heart disease (CHD); myocardial revascularization; risk factors; prognosis.

Abbreviations: LVEDD, left ventricular end-diastolic dimension; LVESD, left ventricular end-systolic dimension; LVEDV, left ventricular end-diastolic volume; LVESV, left ventricular end-systolic volume; IVST, interventricular septal thickness; LVPWT, left ventricular posterior wall thickness; LVMI, left ventricular mass index; LVEF, left ventricular ejection fraction; GFR, glomerular filtration rate; MAU, microalbuminuria.

\section{Introduction}

Prediction of the risk of cardiovascular complications is a priority for preventive medicine, cardiology, and intensive care $[1,2]$. This is due to the severe consequences of diseases

*Corresponding author: Ekaterina S. Levitskaya, PhD. Rostov State Medical University, Rostov-on-Don, Russia. E-mail: es.med@mail.ru of the cardiovascular system that occur in connection with structural adjustment and persistent dysfunction of the heart and other organs and systems. These consequences are caused by many pathological mechanisms, including hyperactivity of neurohumoral systems. Cardiac and renal disorders are closely correlated with formation of a vicious circle that leads to progressive worsening of a patient's prognosis. Cardiorenal continuum plays an important role in the prediction of cardiovascular and renal survival $[3,4]$. In many clinical 
studies, decreasing of renal function was found in patients with arterial hypertension [5] and chronic heart failure [6]; however, the existence of mutually reinforcing pathogenetic relations has been less studied in coronary heart disease (CHD).

Patients with coronary artery disease undergoing coronary reperfusion are a cohort with the highest cardiovascular risk. Operations to restore coronary blood flow are a paramount method of treatment, improving the quality of life in CHD patients. Reducing risk of death and cardiovascular complications is also the purpose of myocardial revascularization (MR). This target is feasible only for a short period of time because mechanical correction of myocardial ischemia is not a means of regulating the pathogenetic mechanisms of CHD progression. The occurrence of restenosis of the coronary arteries and deterioration of cardiovascular prognosis depends on two main factors - the compliance of the patient with optimal medical therapy and the factor environment of the patient. Study of the effect of risk factors (RFs), including markers of renal dysfunction, on the longterm cardiovascular prognosis of patients undergoing surgical MR is a priority for secondary prevention in this cohort of patients. Risk stratification of cardiovascular complications, including cardiovascular death (CVD), is designed to assess the contribution of RFs according to their importance in the formation of the prognosis and to determine the tactics of their correction.

The aim of the present study was to analyze the impact of traditional and renal RFs on the probability of CVD in CHD patients in the remote period after MR.

\section{Materials and Methods}

The present study included 90 CHD patients (80 men and 10 women; mean age $56.1 \pm 0.9$ years) with indications for restoration of coronary blood flow. Coronary angiography was performed in all patients to determine the presence and the degree of luminal obstruction of the coronary arteries and to assess the feasibility and appropriateness of various forms of therapy, such as revascularization by percutaneous or surgical interventions. Data of coronary angiography are presented in Table 1.

\section{Table 1.}

Coronary angiographic parameters in CHD with indications $M R$

\begin{tabular}{|l|c|}
\hline \multicolumn{1}{|c|}{ Variable } & Value \\
\hline Coronary atherosclerosis, number of arteries & $2.06 \pm 0.09$ \\
\hline Subtotal CAS, number of arteries & $2.03 \pm 0.1$ \\
\hline Hemodynamically insignificant CAS, number of arteries & $0.1 \pm 0.03$ \\
\hline Occluded coronary arteries, number of arteries & $0.4 \pm 0.06$ \\
\hline Clinically significant CAS, number of arteries & $2.7 \pm 0.2$ \\
\hline Maximal coronary artery stenosis, \% & $89.1 \pm 1.04$ \\
\hline
\end{tabular}

CAS- Coronary artery stenosis

Coronary artery bypass grafting $(\mathrm{CABG})$ was conducted in $64 / 57.6 \%$ patients and percutaneous coronary intervention (PCI) with stent implantation in $26 / 42.4 \%$ patients. At the beginning of the study, we assessed the prevalence of major cardiovascular RFs: old age, gender, duration of CHD, arterial hypertension $(\mathrm{AH})$, diabetes mellitus (DM), another localization of atherosclerotic lesions, and the presence and duration of smoking. We then estimated the main echocardiographic parameters (LVEDD, LVESD, LVEDV, LVESV, IVST, LVPWT, LVMI, and LVEF) and the parameters of renal function - MAU and GFR. MAU (urinary albumin excretion of 30-300 mg/24 hours) was assessed by a semiquantitative method using test strips for the determination of protein in the urine, in compliance with the rules for collecting morning urine. GFR was estimated by the Cockcroft-Gault formula. Stages of chronic kidney disease (CKD) were determined according to the KDOQI 2002 classification. Patients with CKD stages 4-5 were excluded from this study.

Table 2 presents the data on the prevalence of the studied RFs and the severity of the clinical parameters of the patients.

Table 2.

Traditional cardiovascular RFs and clinical status of CHD patients

\begin{tabular}{|l|c|}
\hline \multicolumn{1}{|c|}{ Variable } & Value \\
\hline Age, years & $56.1 \pm 0.9$ \\
\hline Patients with excess body weight, abs (\%) & $34(37.8)$ \\
\hline Smoking patients, abs (\%) & $32(35.6)$ \\
\hline Total cholesterol, mmol/1 & $5.65 \pm 0.15$ \\
\hline High-density lipoprotein, mmol/1 & $1.1 \pm 0.03$ \\
\hline Low-density lipoproteins, mmol/1 & $4.6 \pm 0.2$ \\
\hline Triglycerides, mmol/1 & $1.95 \pm 0.1$ \\
\hline Dyslipidemia, type IIa, abs (\%) & $47(52.2)$ \\
\hline Dyslipidemia, type IIb, abs (\%) & $29(32.2)$ \\
\hline LVEDV, ml & $151.2 \pm 3.9$ \\
\hline LVESV, ml & $72.3 \pm 3.01$ \\
\hline LVEF, \% & $53.0 \pm 0.7$ \\
\hline IVST, mm & $12.53 \pm 0.17$ \\
\hline LVPWT, mm & $11.99 \pm 0.15$ \\
\hline Angina pectoris class II, abs (\%) & $5(5.6)$ \\
\hline Angina pectoris class III, abs (\%) & $73(81.1)$ \\
\hline Angina pectoris class IV, abs (\%) & $1(1.1)$ \\
\hline Unstable angina, abs (\%) & $8(8.9)$ \\
\hline Acute myocardial infarction, abs (\%) & $3(3.3)$ \\
\hline Myocardial infarction, abs (\%) & $66(73.3)$ \\
\hline Arterial hypertension, abs (\%) & $77(85,6)$ \\
\hline Diabetes, abs (\%) & $19(21.1)$ \\
\hline Atherosclerosis of the lower limb arteries, abs (\%) & $7(7.8)$ \\
\hline Atherosclerosis of the cerebral arteries, abs (\%) & $4(4.4)$ \\
\hline MAU, mg/24 hours & $0.15 \pm 0.02$ \\
\hline The presence of MAU, abs (\%) & $82(91.1)$ \\
\hline GFR, ml/min & $90.2 \pm 2.2$ \\
\hline The presence of CKD, abs (\%) & $88(97.8)$ \\
\hline CKD stage 1, abs (\%) & $48(54.5)$ \\
\hline CKD stage 2, abs (\%) $\%(37.0)$ \\
\hline CKD stage 3, abs (\%) $\%$ \\
\hline
\end{tabular}

The study was conducted in accordance with ethical principles of the Declaration of Helsinki and approved by the Rostov State Medical University Ethics Committee. Written informed consent was obtained from all participants. 
The study included 4 stages. Stage 1: determining the presence and severity of traditional/renal RFs, as well as the severity of the clinical condition of patients. Stages 2, 3, and 4: monitoring of existing RFs and evaluation of the clinical status of the patients on $5.9 \pm 0.2$ days, $6.3 \pm 0.1$ months, and $5.8 \pm 0.05$ years after MR, respectively. In the remote period after MR, the number of patients participating in the fourth phase of the study amounted to $71 / 78.9 \%$. Fatal cardiovascular outcomes were the only endpoint of the study. CVD occurred in $10 / 12.3 \%$ patients.

Statistical analysis of data was performed using the software Statistica 8.0. The mean (M) and standard deviation (SD) were calculated. Differences of continuous variables with a normal distribution between the two groups were calculated using the independent-sample $t$-test. Group comparisons with respect to categorical variables are performed using chi-square tests. Two-tailed $P$ values $<0.05$ were considered statistically significant.

\section{Results}

We determined that the studied RFs, such as the patient's age, duration of smoking, and presence of angina with low tolerance to physical stress, had a significant impact on the probability of death in CHD patients. According to logistic regression analysis (LRA), the risk of cardiovascular death (rCVD) in the remote period after MR in patients of the 35-yearold age group at the time of the MR is minimal and amounts to $0.9 \%$; rCVD is $2.4 \%$ to $6.3 \%$ in the 45 -to- 55 year age group and increases significantly up to $15.4 \%$ to $33.2 \%$ in the 65 -to75 year age group $(P=0.02)$. Addiction to smoking is one of the most common and significant factors of cardiovascular risk. We have found that smoking has a significant impact on rCVD in the remote period after MR. A ten-year smoking history leads to an increase in rCVD up to $5.7 \%$; a 20 -year history, up to $8.3 \%$; a 30 -year history, up to $11.9 \%$; and a 50 -year history, up to $23.1 \%(P=0.04)$. LRA demonstrated that rCVD over a long-term period increases by $18.1 \%$ in patients with elevated levels of total cholesterol determined before revascularization compared with patients who achieved target values of lipid parameters $(P=0.03)$. After $5.8 \pm 0.05$ years following MR, rCVD is increased by $16.2 \%$ in patients with stable angina pectoris class III (CCS) compared with patients with better exercise tolerance $(P=0.04)$. Atherosclerotic lesions of lower limb and cerebral arteries increases rCVD in the remote period after MR by $50.5 \%(P=0.02)$, whereas patients suffering from overweight have rCVD amounting to $69.3 \%(P=0.02)$. Type 2 DM showed no significant deterioration of the cardiovascular prognosis in the long term after MR when taking into account only the fact of DM presence $(P=0.02)$. However, LRA allowed us to demonstrate the important role of a highly reliable level of glucose in the urine for the risk score. When glucosuria is $0.2 \mathrm{~g} / \mathrm{l}, \mathrm{rCVD}$ is $17.6 \%$, whereas it increases by $43 \%(P=0.005)$ with increasing glycosuria up to $0.8 \mathrm{~g} / \mathrm{l}$. The relationship between glycosuria levels and rCVS of CHD patients in the remote period after MR is presented in Figure 1.

Left atrial size $(P=0.02)$, LVPWT $(P=0.02)$, and LVMI $(P=0.02)$ were also significant predictors of adverse cardiovascular prognosis. Risk of CVD in the remote period after MR according to echocardiographic parameters is presented in Table 3.

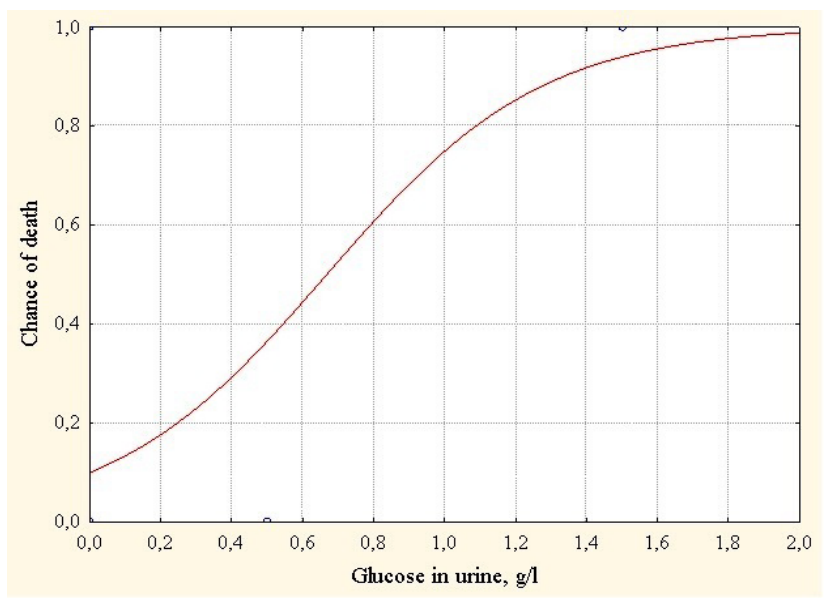

Fig 1. The probability of CVD in CHD patients in the remote period after MR according to the level of glucose in the urine

Table 3

rCVD in the remote period after MR according to echocardiographic parameters

\begin{tabular}{|c|c|c|c|c|c|c|}
\hline & \multicolumn{6}{|c|}{ Left atrial size, $\mathrm{mm}$} \\
\hline & 20 & 30 & 40 & 50 & & 60 \\
\hline \multirow[t]{3}{*}{$\begin{array}{l}\text { Chance of } \\
\text { death, \% }\end{array}$} & 0.4 & 2.4 & 10.8 & 37.5 & & 74.8 \\
\hline & \multicolumn{6}{|c|}{ LVPWT, mm } \\
\hline & 9 & 11 & 13 & 15 & & 17 \\
\hline \multirow[t]{3}{*}{$\begin{array}{l}\text { Chance of } \\
\text { death, \% }\end{array}$} & 1.8 & 5.3 & 14.3 & 33.4 & & 60.1 \\
\hline & \multicolumn{6}{|c|}{ LVMI, $\mathrm{g} / \mathrm{cm}^{3}$} \\
\hline & 140 & 180 & 220 & 260 & 300 & 340 \\
\hline $\begin{array}{l}\text { Chance of } \\
\text { death, \% }\end{array}$ & 8.0 & 16.2 & 30.2 & 49.0 & 68.1 & 82.6 \\
\hline
\end{tabular}

Analysis of renal RFs allowed us to establish a high prevalence of renal dysfunction in CHD patients with indications for MR. The prevalence of MAU amounted to $91.1 \%$ of cases with an average level of $110 \pm 7 \mathrm{mg} /$ day; the incidence of CKD amounted to $97.8 \%$ of cases. We have not found a significant impact of MAU on rCVD. However, LRA revealed a highly significant influence of other indicators of renal dysfunction on rCVD. The presence of CKD stage 3 increases $\mathrm{rCVD}$ in the period before MR $(58.8 \% ; P<0.001)$ and in the late period (6.3 \pm 0.1 months) after MR $(27.9 \%$; $P=0.02)$. The impaired GFR, defined later, $5.9 \pm 0.2$ days after MR significantly increases $\operatorname{rCVD}(P=0.04)$ (Fig.2).

We studied also the characteristics of coronary lesions. The increase in the number of coronary arteries with clinically significant stenosis $(P=0.03)$, including subtotal narrowing of the vessel lumen $(P=0.03)$, increases $\mathrm{rCVD}$ in the long-term period. Stratification of rCVD in the remote period after MR is presented in Table 4. Obviously, the increased rCVD in CHD 
patients occurs when the severity of myocardial ischemia is exacerbated due to the number of atherosclerotic-affected coronary arteries and the degree of stenosis.

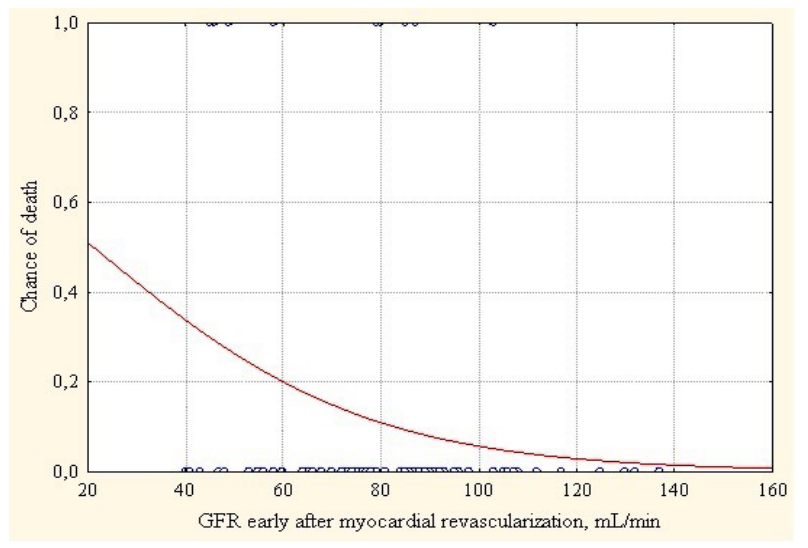

Fig.2. The probability of CVD in CHD patients in the remote period after MR according to the value of GFR

Table 4.

The probability of $C V D$ in $C H D$ patients in the remote period after MR according to the severity of coronary lesions

\begin{tabular}{|c|c|c|c|c|c|c|}
\hline \multirow{2}{*}{ Chance of death, \% } & \multicolumn{6}{|c|}{ Clinically significant CAS, number of arteries } \\
\cline { 2 - 7 } & 1 & 2 & 3 & 4 & 5 & 6 \\
\hline & 5.3 & 7.8 & 11.4 & 16.4 & 22.9 & 31.1 \\
\cline { 2 - 7 } & 1 & 2 & 3 & 4 & 5 & 6 \\
\hline Chance of death, \% & 6.3 & 10.3 & 16.4 & 25.2 & 36.6 & 49.8 \\
\hline
\end{tabular}

\section{Discussion}

The importance of predicting the risk of cardiovascular complications is determined by the high prevalence of cardiovascular diseases, mortality, and the reduced quality of life of patients suffering from cardiovascular diseases [7]. According to the latest WHO report (Inf. Bulletin No. 317, Jan 2015), 17.5 million people died from cardiovascular diseases in 2012, which corresponds to $31 \%$ of all causes of death in the world. It is important that 14.1 million deaths were caused by CHD and acute cerebral accidents with the primary contribution of myocardial ischemia to the mortality structure. Patients included in our study had a high cardiovascular risk because of a large prevalence of traditional and renal RFs. Every third patient had a history of being overweight addicted to smoking, and almost all patients did not achieve target levels of cholesterol. We registered cardiovascular accidents in patient histories and the presence of hypertension in the most cases. The severity of the clinical condition and a large number of RFs explain the high mortality rate in the remote period after MR.

The age of CHD patients is a well-known RF of the disease progression. According to H. Ao [8], the risk of death after having coronary reperfusion performed significantly increased in the older cohort of patients compared with younger patients. Our study also demonstrated the great importance of assessing the patient's age. Smoking is the most well-known predictor of cardiovascular complications [9]. The results of many studies indicate that a poor prognosis for cardiovascular diseases depends not only on smoking, but also on the duration of nicotine dependence. According to a study by L.S. Rallidis [10] conducted on a cohort of young patients aged less than 35 years who have had an acute MI, it has been shown that there is a significant increase in risk of cardiovascular events (death, acute coronary syndrome, revascularization) with increased duration of smoking. At the same time, some studies have demonstrated conflicting evidence. A study conducted on a group of 8,671 CHD patients with MR shows that there were no significant differences in the incidences of angiographic restenosis between groups of patients who smoked and those who had no nicotine dependence, whereas the clinical manifestations of myocardial ischemia were more often registered in non-smoking patients [11]. The authors of the study attribute the differences to the decreased sensitivity to myocardial ischemia in smoking patients and a reluctance to consult a doctor in cases of recurrent angina. The results of other studies indicate an increasing probability of death and acute MI after coronary reperfusion in patients with a long history of smoking $[9,12]$; quitting smoking before or after revascularization significantly reduced the risk of adverse cardiovascular complications in study by T.Chen [9]. The analysis of present study confirms the adverse effect of smoking duration on cardiovascular prognosis and demonstrates its impact on mortality in the remote period after MR.

The relationship between the severity and prevalence of myocardial ischemia and an increased risk of cardiovascular complications is not in doubt [13]. We have shown that rCVD in the remote period after MR increases with an increase in the number of the atherosclerotic affected arteries with clinically significant stenosis, including subtotal stenosis. The presented data on the increase in the probability of death in the remote period in patients with peripheral atherosclerosis and ischemic disease of the brain fit into the concept of atherosclerosis as a generalized process.

Mechanisms that regulate glucose homeostasis in the kidneys are controlled primarily by transport proteins engaged in the reabsorption of glucose in the proximal renal tubules. It is known that there is a complete return absorption of glucose up to its absence in the secondary urine under physiological conditions. The system of protein transport carries out the glucose reabsorption even under conditions of hyperglycemia. Depletion of protein transporters with the subsequent glycosuria occurs when the threshold concentration of blood glucose is greater than $10 \mathrm{mmol} / 1$ (180 mg/dl) [14]. Many studies on the pathological influence of uncontrolled hyperglycemia exhibit an increased risk of developing macroand microvascular complications [14]. We found that the likelihood of increased risk of death in the remote period after MR depends on an increase in the urine glucose level, while there were no data indicating increased rCVD in DM patients.

Echocardiographically determined left atrial size has been shown to be a significant predictor of cardiovascular 
complications in patients with various cardiovascular diseases [15]. The increased left atrial size is a marker of adverse cardiovascular prognosis in patients with atrial fibrillation [16], acute MI [17], in elderly patients with or without cardiac arrhythmias [18], and heart failure [16]. Many studies, including ours, have identified an association between the increased left atrial size and an increase in the probability of death in patients suffering from myocardial ischemia. The results can be explained by the known data indicating that the increased left atrium is closely associated with overproduction of atrial natriuretic peptide (ANUP) that is an independent predictor of cardiovascular complications. We also found that myocardial ischemia is one of the factors causing the increase in ANUP formation as a result of myocardial remodeling that occurs in response to ischemia [19].

Myocardial hypertrophy is one of the important echocardiography markers in the cardiovascular risk stratification. The increased LVMI and left ventricular wall thickness, emerging due to high blood pressure, increases the risk of cardiovascular death, as shown in the famous Framingham Heart Study [20]. In our study, we have also shown that left ventricular hypertrophy affects the risk of death for patients in the remote period after MR.

Currently, an identification of renal RFs is one of the priority positions in the research finding. It is a proven fact that renal dysfunction has an impact on the risk of cardiovascular complications in patients with socially significant cardiac pathology. Our earlier research has demonstrated the high importance of the magnitude of MAU. The risk of coronary events has been increased by $5 \%$ with a 2 -fold increase in the minimum MAU level in patients before coronary intervention; the risk increased further by $7 \%$ at maximum MAU level [21]. The risk of recurrent angina in patients with CKD stage 3 was significantly higher in the late period ( $6.3 \pm 0.1$ months $)$ after MR [22]. We have found the great contribution of reduced GFR on rCVD in patients undergoing surgical MR. The reliability of the obtained data was established for CKD stage 3, which was shown for the initial period before coronary reperfusion and in the late period of surgical intervention. The obtained data confirm the importance of determining markers of renal dysfunction as reliable predictors of CHD progression and the selection of the group of patients with a high probability of dying in the remote period after revascularization. There is a dependence of rCVD on GFR values, which is determined in a short period after MR. The frequency of having to perform acute dialysis after surgical interventions on the heart is from $1 \%$ to $5 \%$ of cases [23[. Mechanisms of kidney injury in cardiac surgery are multifactorial and include a number of key conditions - low cardiac output, hypoperfusion and ischemia of the kidney tissue, and loss of pulsating flow of blood circulation [24]. M.L. Felicio et al. [24] showed that a significant decrease in GFR occurs in patients after coronary artery bypass surgery. We have also demonstrated that a significant decrease in GFR is observed in the early period after MR and after $6.3 \pm 0.1$ months after coronary reperfusion compared with the period before MR. The decrease of GFR in the short and long period after MR can be interpreted as a decrease in the functionality of the renal tissue in conditions of hemodynamic imbalance. The degree of GFR reduction indicates the severity of impairments in the heart-kidney relationships and determines the prognosis of cardiovascular complications.

\section{Conclusion}

Data obtained from our study demonstrate a high medical and social importance of a comprehensive and integrated analysis not only of traditional RFs, but also of markers of renal dysfunction in risk stratification of cardiovascular prognosis in CHD patients. A comprehensive analysis of RFs should be performed on every CHD patient with indications for restoration of coronary blood flow. This will help optimize the clinical condition of the patient and timely correction of existing RFs.

\section{Competing interests}

The authors declare that they have no competing interests.

\section{Acknowledgment}

V. Chistyakov was supported by grant $6.1202 .2014 / \mathrm{K}$ from the Ministry of Education and Science of the Russian Federation.

\section{References}

1. Hussain SM, Oldenburg B, Wang Y, Zoungas S, Tonkin AM. Assessment of Cardiovascular Disease Risk in South Asian Populations. Int J Vasc Med. 2013; 2013:786801. doi: 10.1155/2013/786801.

2. Laffey JG, Talmor D. Predicting the development of acute respiratory distress syndrome: searching for the "Troponin of ARDS". Am J Respir Crit Care Med. 2013; 187(7):671-2.

3. García-Donaire JA, Ruilope LM. Cardiovascular and Renal Links along the Cardiorenal Continuum. Int J Nephrol. 2011; 2011:975782. doi: 10.4061/2011/975782.

4. Slee AD. Exploring metabolic dysfunction in chronic kidney disease. Nutr Metab (Lond) 2012; 9(1):36. doi: 10.1186/1743-7075-9-36.

5. Osuji CU, Nwaneli CU, Onwubere BJ, Onwubuya EI, Ahaneku GI. Renal function in patients with hypertension associated congestive cardiac failure seen in a tertiary hospital. Int J Nephrol. 2012; 2012:769103. doi: 10.1155/2012/769103. 6. Smith GL, Shlipak MG, Havranek EP, Masoudi FA, WM McClellan, Foody JM, et al. Race and renal impairment in heart failure: mortality in blacks versus whites. Circulation. 2005; 111(10):1270-7.

7. Mozaffarian D, Benjamin EJ, Go AS, Arnett DK, Blaha MJ, Cushman M,et al. Heart disease and stroke statistics 2015 update: a report from the American Heart Association. Circulation. 2015; 131(4):e29-322.

8. Ao H, Wang X, Xu F, Zheng Z, Chen M, Li L, et al. The impact of body mass index on short- and long-term outcomes in patients undergoing coronary artery graft bypass. PLoS One. 2014; 9(4):e95223. doi: 10.1371/journal.pone.0095223. 9. Chen T, Li W, Wang Y, Xu B, Guo J. Smoking status on outcomes after percutaneous coronary intervention. Clin 
Cardiol. 2012; 35(9):570-4.

10. Rallidis LS, Lekakis J, Panagiotakos D, Fountoulaki K, Komporozos C, Apostolou T, et.al. Long-term prognostic factors of young patients ( $<$ or $=35$ years) having acute myocardial infarction: the detrimental role of continuation of smoking. Eur J Cardiovasc Prev Rehabil. 2008; 15(5):567-71. 11. Cohen DJ, Doucet M, Cutlip DE, Ho KKL, Popma JJ, Kuntz RE, et al. Impact of smoking on clinical and angiographic restenosis after percutaneous coronary intervention: another smoker's paradox? Circulation. 2001; 104(7):773-8.

12. Sherif MA, Nienaber CA, Toelg R, Abdel-Wahab M, Geist V, Schneider S, et al. Impact of smoking on the outcome of patients treated with drug-eluting stents: 1-year results from the prospective multicentre German Drug-Eluting Stent Registry (DES.DE). Clin Res Cardiol. 2011; 100(5):413-23.

13. Aldweib N, Negishi K, Hachamovitch R, Jaber WA, Seicean S, Marwick TH. Impact of repeat myocardial revascularization in silent ischemia after previous revascularization. J Am Coll Cardiol. 2013; 61(15):1616-23.

14. Curtis LT. Understanding the kidneys' role in blood glucose regulation. Am J Manag Care. 2012; 18(1):11-6.

15. Teresa SMT, Walter PA, Marion EB, Miyasaka Y, Gersh B J, Bailey KR, et al. Prediction of cardiovascular outcomes with left atrial size : is volume superior to area or diameter? J Am Coll Cardiol. 2006; 47(5):1018-23.

16. Tsang TS, Barnes ME, Gersh BJ, Bailey KR, Seward JB. Risks for atrial fibrillation and congestive heart failure in patients $>/=65$ years of age with abnormal left ventricular diastolic relaxation. Am J Cardiol. 2004; 93(1):54-8.

17. Moller JE, Hillis GS, Oh JK, Seward JB, Reeder GS, Wright RS, et al. Left atrial volume: a powerful predictor of survival after acute myocardial infarction Circulation.
2003; 107(17):2207-12.

18. Barnes ME, Miyasaka Y, Seward JB, Gersh BJ, Rosales $\mathrm{AG}$, Bailey KR, et al. Left atrial volume in the prediction of first ischemic stroke in an elderly cohort without atrial fibrillation. Mayo Clin Proc. 2004; 79:1008-14.

19. Bergeron S, Møller JE, Bailey KR, Horng HC; John C B. Pellikka PA. Exertional changes in circulating cardiac natriuretic peptides in patients with suggested coronary artery disease. J Am Soc Echocardiogr. 2006; 19(6):772-6.

20. Bouzas-Mosquera A, Broullón FJ, Álvarez-García N, Peteiro J, Mosquera VX, Castro-Beiras A. Association of left ventricular mass with all-cause mortality, myocardial infarction and stroke. PLoS One. 2012; 7(9): e45570. doi: 10.1371/journal.pone.0045570.

21. Batiushin MM, Levickaja ES, Terent'ev VP, Savis'ko AA, Djuzhikov AA, Kulikovskih JaV. Renal and coronary predictors of post-myocardial revascularization prognosis in patients with coronary heart disease. Russ J Cardiol. 2012; 6(98):45-50. [Article in Russian].

22. Batiushin MM, Levickaja ES, Terent'ev VP, Djuzhikov AA, Poddubnyj AV. Predicting cardiovascular risk in patients with coronary artery disease who underwent myocardial revascularization, taking into account the presence of renal and cardiovascular risk factors. Klin Nefrol. 2011; 1:39-42. [Article in Russian].

23. Bahar I, Akgul A, Ozatik MA, Vural KM, Demirbag AE, Boran M, et al. Acute renal failure following open heart surgery: risk factors and prognosis. Perfusion. 2005;20(6):317-22. 24. Felicio ML, Andrade RR, Castiglia YM, Silva MA, Vianna PT, Martins AS. Cystatin C and glomerular filtration rate in the cardiac surgery with cardiopulmonary bypass. Rev Bras Cir Cardiovasc. 2009; 24(3):305-11. 